\title{
Research of Huangguogan anthracnose infection time and optimum control period
}

\author{
Yinghuan $\mathrm{Xu}^{1, \text { a }}$, Zhihui Wang ${ }^{2^{*}}$ and Bo Xiong ${ }^{3}$ \\ ${ }^{1}$ College of Horticulture, Sichuan Agricultural University, Sichuan, China \\ ${ }^{2}$ Institute of Pomology and Olericulture, College of Horticulture, Sichuan Agricultural University, \\ Sichuan, China \\ ${ }^{3}$ College of Horticulture, Sichuan Agricultural University, Sichuan, China \\ aHannahSAU@163.com
}

Keywords: Huangguogan; anthracnose; infection; optimum control period

\begin{abstract}
This paper studies the Huangguogan anthracnose infected time and different concentration of 25\% Pyraclostrobin EC (1500, 2000, 2500 times) in Huangguogan in different periods (before flowering, after flowering, young fruit period, fruit rapid growth period) control effect on Huangguogan anthracnose after application. The results showed that the best control effect on anthracnose of Huangguogan was after anthesis. Among them, the control effect of 2000 times $25 \%$ Pyraclostrobin EC was the best, and the control effect reached 53\%. However, the control effect of 2500 times $25 \%$ Pyraclostrobin EC before flowering was the worst only $21.4 \%$. Therefore, through the comprehensive analysis of field control results obtained after flowering for the prevention and treatment of Huangguogan anthracnose period is the optimum control period. Huangguogan anthracnose infected time during after flowering to fruit period.
\end{abstract}

\section{Introduction}

Citrus belong to the Rutaceae family and Citrinae [1]. It started about 30 million years ago [2]. Huangguogan is a unique and resource-rich orange and orange natural hybrids in Sichuan [3]. It is a kind of new variety with independent intellectual property rights in Sichuan province. It has many advantages, like late maturing, special high yield, non-nuclear, sweet and sour moderate, etc [4]. Anthrax is a fungal disease that causes bacteria to spread by wind, rain and dew. It is easy to disease when high temperature and high humidity, less spring, summer and autumn as much [5]. Through observations and experiments revealed that a large number of Huangguogan in Shimian county were infected with one kind of pathogenic bacteria, C. gloeosporioides. The amount of latent infection before the fruit harvest and post-harvest disease severity are closely linked [6]. Citrus anthracnose can lurk on citrus flowers and the surface of fruits [7]. However, it remains unclear whether the fruit has been lurched and harmed other parts before fruit development.

\section{Materlals and Methods}

Experimental Location, Plants and Reagents. The trial was conducted at Anshun field, Shimian county, Sichuan province. The experimental plants were 7 years old, tree crown diameter of the same, perennial infected with anthracnose Huangguogan trees. Among them, 12 trees treated with water was used as control and the observation of the beginning period of infection. There were 36 tree species that were treated with potion. The experimental plants were $25 \%$ Pyraclostrobin (EC) 1500 times, 25\% Pyraclostrobin (EC) 2000 times, 25\% Pyraclostrobin (EC) 2500 times.

Experimental Treatment. The 12 observation trees were observed from the early flowering stage. Observation of early flowering were shoots, leaves; Flowering observed objects were shoots, leaves, flowers (stigma); Observations of young fruit objects were branches, leaves, fruits; The rapid growth of fruit observed objects were leaves, branches, fruits. In the observation process recorded the observation time, the affected part and its disease characteristics. Spraying experimental drugs were spent in the pre-flowering, post-flowering, young fruit, fruit rapid growth period. 
Experimental treatment used randomized block design. Single plant was a district. Experiment set 3 concentration treatment. Each treatment set 3 repetitions. Experimental set water as a control. The experiment was administered once per period with tree crown spray method.

Control Effect Determination. Each tree was divided into four directions: east, south, west and north. Each position was randomly surveyed from inside the hall and outside. Each position was randomly surveyed from inside the hall and outside. The experiment selected 100 fruits to calculate the disease index and the control effect. Efficacy calculation method refer with: Eq.1, Eq.2.

$$
\begin{aligned}
& \text { Disease index }=\frac{\sum(\text { Number of diseased fruit } * \text { Number of representatives at that level })}{\text { Total number of fruits surveyed } * \text { The highest representation of disease }} * 100 \% \\
& \text { Relative control effect }=\frac{\text { Control disease index-Treatment index }}{\text { Control disease index }} * 100 \%
\end{aligned}
$$

Citrus peel lesions classification method is formulated as follows ( 0 belongs to the normal fruit):

0 level: no disease;

1 level: Small spots and spots should not be analyzed. The lesion area occupies less than $5 \%$ of the whole fruit area;

3 level: Lesions easy to identify. It has affected the commerciality of the fruit. The lesion area occupies $5 \%$ to $10 \%$ of the whole fruit area;

5 level: The lesion area occupies $11 \%$ to $25 \%$ of the whole fruit area. It has obviously affected the commerciality of the fruit;

7 level: The lesion area occupies $26 \%$ to $50 \%$ of the whole fruit area. It seriously affects the commerciality of the fruit;

9 level: The lesion area occupies more than $50 \%$ of the whole fruit area. Fruit product completely declined.

\section{Results and Analysis}

Infection Period Investigation. Table 1 shows that anthracnose infection of the Huangguogan period is in the young fruit period. It infects during flowering to young fruit period. Infected characteristics first manifested in the Huangguogan leaves.

Table 1 anthracnose infection time survey

\begin{tabular}{llll}
\hline Observation period & Observation site & feature & Observation date \\
\hline pre-flowering & Leaf & No abnormality & 2016.3 .22 \\
& Branches & No abnormality & 2016.3 .22 \\
Flowering period & Leaf & No abnormality & 2016.4 .6 \\
& Branches & No abnormality & 2016.4 .6 \\
& flower & No abnormality & 2016.4 .6 \\
young fruit period & Leaf & Withered dehydration, curly shape & 2016.4 .26 \\
& Branches & No abnormality & 2016.4 .26 \\
& fruit & No abnormality & 2016.4 .26 \\
fruit rapid growth period & Leaf & Edges or near the edges appear brown & 2016.5 .10 \\
& & spots, or was curly shape & 2016.5 .10 \\
& Branches & No abnormality & 2016.5 .10 \\
\hline
\end{tabular}

Control Effect. Table 2 shows that pre-flowering, post-flowering, young fruit and fruit rapid growth period were applied 25\% EC 1500 times, 2000 times, 2500 times for Huangguogan anthracnose had a certain control effect. As the interaction between the control period and the treatment concentration had no significant effect on the control effect. Therefore, the application period and the drug concentration were analyzed. 
Table 2 Huangguogan anthracnose prevention and control statistics

\begin{tabular}{lccc}
\hline Drug spraying period & 1500 times & 2000 times & 2500 times \\
\hline pre-flowering & 39.2 & 47.8 & 21.7 \\
& 21.7 & 47.8 & 13.1 \\
& 21.7 & 21.7 & 29.2 \\
post-flowering & 58.2 & 58.2 & 37.3 \\
& 53.0 & 47.8 & 36.6 \\
young fruit period & 36.6 & 53.0 & 40.2 \\
& 21.7 & 47.8 & 47.8 \\
& 30.3 & 39.2 & 21.7 \\
fruit rapid growth period & 25.8 & 47.8 & 30.3 \\
& 32.1 & 39.2 & 37.3 \\
& 25.3 & 30.3 & 16.4 \\
\hline
\end{tabular}

Effects of Anthracnose in Huangguogan during Different Pharmaceutics Treatment. Table 3 shows that applying $25 \%$ EC reagent in four periods all had some control effect on anthracnose of Huangguogan. The effect of pesticides in the post-flowering was the best, reaching $46.8 \%$. The effect of pesticides in the rapid growing period of fruits was the worst, reaching only $26.7 \%$.

Table 3 different periods of drug treatment on the prevention and treatment of Huangguogan anthrax

\begin{tabular}{lll}
\hline time & Disease index & Control effect $(\%)$ \\
\hline pre-flowering & $27.1 \mathrm{~b}$ & $29.3 \mathrm{~b}$ \\
post-flowering & $20.4 \mathrm{c}$ & $46.8 \mathrm{a}$ \\
young fruit period & $25.0 \mathrm{bc}$ & $35.3 \mathrm{~b}$ \\
fruit rapid growth period & $28.2 \mathrm{~b}$ & $26.7 \mathrm{~b}$ \\
CK & $38.3 \mathrm{a}$ & --- \\
\hline
\end{tabular}
below.

Note: Lowercase letters indicate significant differences between treatments $(\mathrm{P}<0.05)$, the same

Effect of Different Concentrations of 25\% EC on Anthracnose Control of Huangguogan. Table 4 shows that $25 \%$ EC for the prevention and treatment of Huangguogan anthrax had a certain effect. It was best to control the anthracnose of Huangguogan by $25 \%$ EC2000, reaching $41.9 \%$. The liquid control effect of the $25 \%$ EC 1500 times and 2500 times was less than the control effect of the 2000 times which difference is not obvious. The results showed that $25 \% \mathrm{EC}$ in this experiment for anthracnose prevention and treatment of Huangguogan optimum concentration was about 2000 times.

Table 4 different concentrations of $25 \% \mathrm{EC}$ on the prevention and treatment of Huangguogan anthrax

\begin{tabular}{lll}
\hline Processing concentration & Disease index & Control effect $(\%)$ \\
\hline $25 \%$ Pyraclostrobin (EC)1500 & $26.2 \mathrm{~b}$ & $31.5 \mathrm{~b}$ \\
25\% Pyraclostrobin (EC)2000 & $22.2 \mathrm{c}$ & $41.9 \mathrm{a}$ \\
$25 \%$ Pyraclostrobin (EC)2500 & $27.0 \mathrm{~b}$ & $29.4 \mathrm{~b}$ \\
CK & $38.3 \mathrm{a}$ & --- \\
\hline
\end{tabular}

\section{Summary}

The results of this experiment show that there was no surface feature of anthracnose infestation before flowering and flowering period of Huangguogan. Huangguogan anthrax infection period was the beginning of young fruit period. Infestation begins after florescence until young fruit period. This is similar to the conclusion drawn by S.X. Liu [9] on the effect of bagging on anthracnose disease of apple blight in Fuji apple. After flowering was the prevention and treatment of Huangguogan anthrax suitable period. This is similar to the results of Li Xuebin [10] in the research on the occurrence and control of citrus anthracnose. Conclusions were drawn from the control efficacy of three different concentrations of $25 \%$ Pyraclostrobin (EC) used in the experiment. The 
best control effect of Huangguogan is to use 25\% Pyraclostrobin (EC) 2000 times after flowering.

Citrus anthracnose prevention and control strategies should be based on prevention. If the onset of symptoms appeared after the application of pesticide control effect is not ideal. So, after flowering timely chemical control can significantly reduce the risk of anthrax infection.

\section{Acknowledgements}

This experiment is completed under the careful guidance of Z.H. Wang teacher. My teacher gave me a lot of help and support throughout the experiment. I expressed my sincere thanks to my teacher. In addition, thanks to B. Xiong for my answer during the experiment.

\section{References}

[1] W.T. Swingle \& P.C. Reece: The botany of Citrus and its wild relatives (The Citrus Industry, Berkeley 1967).

[2] T. Tanaka \& Citrologia: semi-centennial commemoration papers on citrus studies (Citrologia Supporting Foudation for Tanaka International Citrus Institute, Japan 1961).

[3] Z.Q. Zhang \& D.H. Wang: Study on the genetic relationship of Huangguogan with isozymes (Journal of Sichuan Agricultural University, Sichuan 1994).

[4] Z.H. Wang, S.F. Lu and Q.Q. Yan: Biological Characteristics Survey and the Differences Compare in Strains of Huangguogan in Shimian (Northern Horticulture, Heilongjiang 2011).

[5] J.R. Lei \& Z.X. Fan: Prevention and Cure Measures of Citrus Major Diseases (Chinese Gardening Abstracts, Beijing 2009).

[6] Y.C. Li \& Y. Bi: Occurrence and infection of Alternaria rot of Pingguoli pear, Pyrus pyrifolia (Journal of Plant Protection, Beijing 2006).

[7] W.F. Wang \& H.Q. Leng: Universality and infectivity of latent infection of citrus anthracnose (Journal of Sichuan Agricultural University, Sichuan 1990).

[9] S.X. Liu: Effect of Bagging on Anthracnose Disease of Fuji Apple (Hebei fruits, Hebei 2002).

[10] X.B. Li: Occurrence and Control of Citrus Anthracnose (Chinese Horticulture Abstracts, Beijing 2011). 